\title{
Editorial for JECR special issue on defects \& relaxation processes in crystalline and amorphous solids
}

\author{
Himanshu Jain • Harry L. Tuller
}

Published online: 4 February 2015

(C) Springer Science+Business Media New York 2015

Key physical and chemical properties of functional materials depend largely on the existence of structural and electronic defects within their crystalline lattices or amorphous structure. This special issue on Defects \& Relaxation Processes in Crystalline and Amorphous Solids is dedicated to the late Professor Arthur Stanley Nowick, a pioneer in the modeling and characterization of defect formation and transport by anelastic and dielectric relaxation behavior and ionic conductivity. Professor Nowick made seminal contributions to our understanding of fast oxygen and proton conduction in oxides, alkali metal ion transport in quartz and glasses, diffusion in metals and metal halides, dislocation motion and generation in metals, morphological stability, surface structure and the application of dilatometry and X-ray diffraction for the identification and monitoring of defects in solids. [1] He was highly regarded for the clarity of his writing, having authored two authoritative books in the defects field including, Anelastic Relaxation in Crystalline Solids, (coauthored with Brian S. Berry) in 1972, [2] and Crystal Properties via Group Theory in 1995. [3] His career was split between academia and industry, serving as Assistant/Associate Professor of Metallurgy, Yale Univ. (1951-57) and later as Professor of Metallurgy and Materials Science \& Henry Marion Howe Professor, Columbia Univ. (1966-93). While at Columbia, he received the Great Teacher Award (1987). In the intervening years, he

H. Jain

Department of Materials Science and Engineering, Lehigh

University, Bethlehem, PA 18015, USA

e-mail: h.jain@lehigh.edu

H. L. Tuller $(\square)$

Department of Materials Science and Engineering, Massachusetts Institute of Technololgy, Cambridge, MA 02139, USA

e-mail: tuller@mit.edu served as the Head of the metallurgy group at the IBM T. J. Watson Research Center. Outside of his scientific career, he was an accomplished pianist, with wide-ranging interests in classical music and jazz. An insightful description of his career and accomplishments was published in 2012 by Prof. Daniel N. Beshers, his longtime Columbia University colleague and collaborator [4].

Several Memorial Symposia were held in 2013 in honor of Professor Arthur Stanley Nowick, including a satellite symposium in conjunction with the 19th International Conference on Solid State Ionics, June 7, 2013, in Kyoto, Japan, and special sessions on Ionics and Nano-ionics held during the 7th International Discussion Meeting on Relaxations in Complex Systems, held in Barcelona, July 21-26, 2013. This special issue, dedicated to highlighting the impact of his work on studies of defects and relaxation processes in crystalline and amorphous solids, includes 13 invited and contributed papers from key participants of these two symposia. A previous special issue published in 1994, honoring his 70th birthday, offers a summary of earlier contributions from his former students, colleagues and collaborators. [5].

The special issue begins with an article by Funke et al., entitled Toward understanding the second universality-A journey inspired by Arthur Stanley Nowick. [6] It attempts to explain "the ubiquitous second universality of ac conductivity" or equivalently "the nearly constant loss" behavior of electrical relaxation that was first established by Nowick. [7] The authors recount how a conversation with Nowick helped clarify its distinctive features and subsequently develop an atomistic explanation as elaborated in this article. Díaz-Guillén, et al. [8] investigate this phenomenon in detail in the next article entitled: Nearly constant loss in crystalline oxide-ion conductor $\mathrm{Gd}_{2} \mathrm{Zr}_{2} \mathrm{O}_{7}$. The authors trace the origin of observed behavior in 'jellyfish'-like, collective wiggling motions of 
caged ions that dominate at low temperatures and short times - a view initially developed by Nowick et al. [9] The third article is by Shaw et al. entitled Ion dynamics in single and mixed former glasses: Correlation between microscopic lengths and network structure. [10] It reports on the correlation between frequency dependent ac conductivity and glass structure that is varied systematically by changing composition of several oxide glass systems.

The next two articles focus on dielectric relaxation in complex ferroelectric systems. Firstly, Omari et al. report the results of a 'Study of dielectric relaxation and diffuseness character of sol-gel derived $\mathrm{Pb}_{1-x} \mathrm{La}_{x}\left(\mathrm{Fe}_{0.03} \mathrm{Ti}_{0.97}\right) \mathrm{O}_{3}$ ceramics synthesized at lower temperature for modern nanotechnological devices'. [11] The investigated system is a relaxor ferroelectric titanate ceramic with potential for applications in nanoscale devices. On the other hand, Liu and Zheng discuss Anelastic analyses on the relaxation of antiferroelectric states in $0.94 \mathrm{Bi}_{0.5} \mathrm{Na}_{0.5} \mathrm{TiO}_{3}-0.06 \mathrm{BaTiO}_{3}$ solid solutions under electric fields. [12] A particularly interesting aspect of this work is the observation of mechanical anelastic response under the application of electric field. The simultaneous application of mechanical and electrical fields provides a greater insight of the anti-ferroelectric behavior. It demonstrates the usefulness of the method for characterizing electromechanical or electro-chemical processes of solid state ionics.

The following three papers are dedicated primarily to the long range migration of alkali ions in crystal and glasses. The paper by Habasaki \& Ngai, entitled Molecular dynamics study of heterogeneous dynamics in lithium disilicate crystal, [13] is a theoretical simulation of the atomistics of ion motion in a model system. The authors compare ion dynamics in the crystalline and glassy states of the same material, explaining why ions can move much faster in glasses than corresponding perfect crystals of the same composition. Their simulations yield the nearly constant loss behavior even for a perfect crystal. In this regard, an important message is that 'heterogeneous dynamics of the ions is caused by ion-ion interactions and correlations rather than exclusively from disorder of the network structure'. In the next paper entitled, Effect of tin and gold on sodium ion movement in a sodium silicate glass, [14] Saiyasombat \& Jain report an unexpected result that the presence of metal (gold and/or tin) nanoparticles can significantly enhance the migration of alkali ions in a sodium silicate glass. The work illustrates the importance of polarizability as well as openness of glass network structure as key factors for ion diffusion. The final paper in this section of the special issue, entitled ${ }^{204} \mathrm{Tl}$ tracer diffusion and conductivity in thallium germanium sulphide glasses over a wide composition range by Bokova et al. [15] reports on the dramatic effect of composition on dc conductivity in a chalcogenide glass system. By comparing de conductivity with radiotracer diffusion of thallium ions, the authors show that ionic conductivity dominates for glass with $>15$ at $\% \mathrm{Tl}$. The motion of $\mathrm{Tl}$ ions is found to be uncorrelated up to $\sim 17 \mathrm{at} \% \mathrm{Tl}$, which is an order of magnitude higher concentration than the value for other ionically conducting oxide or sulfide glass systems.

We next switch to the consideration of ionic transport in nanosized particles and films. We begin with an article by Joachim Maier, a pioneer in the field of Nanoionics with a review article entitled Nanoionics: size effects and storage in small systems. [16] Charged defect densities are well known to vary dramatically close to interfaces due to space charge effects. In this article, the author emphasizes the special role of nano-dimensions, for which overlapping space charge regions leave behind no bulk regions. This has important implications for mixed ionic conductors, the modeling of defect equilibria at interfaces, and rapid mass and supercapacitive storage at interfaces in mixed phase systems.

This is followed by an article by Shen et al. on Using thin films to investigate heterogeneous defect chemistry. [17] The authors note the contribution of Nowick towards our understanding of ion transport in compositionally homogeneous materials. In this work. they suggest that the analogy of electronically conducting heterogeneous superlattices can be transferred to the ionic conducting domain thereby enabling examination of both space charge and strain effects on ion transport. The impact of interlayer spacing and composition, dopant type and phase on ionic conduction in ceria and zirconia based electrolytes are analyzed and reported.

The focus on nanosized dimensions and their impact on defects and transport, is next directed towards the study of the unique properties of nominally loose and partially sintered nanosized particles. In the article by Knauth et al., entitled Study of compaction and sintering of nanosized oxide powders by in situ electrical measurements and dilatometry: Nano $\mathrm{CeO}_{2}$ —case study, [18] the authors apply a novel approach to simultaneously monitor the densification and electrical properties of nanosized ceria particles within a modified dilatometer while controlling both temperature and oxygen partial pressure. The key finding, that nanosized particles reduce much more readily than bulk specimens, has potentially important implications for the use of such particles as catalyst supports and in gas sensing devices.

During the Nowick era, only bulk ceramics were considered in the design of solid oxide fuel cells. With the advent of small portable electronic devices, correspondingly small power sources were needed. This has led, in the past decade, to a growing interest in replacing batteries with micro fuel cells promising longer operating times and rapid recharge. [19] Following a more technological focus, Kerman et al. consider Free standing yttria-doped zirconia membranes: Geometrical effects on stability. [20] Rather than bulk three dimensional ionic transport, or even transport in supported thin films, the authors consider instead the implications on ion transport from self supported thin film membranes with ability to buckle under built-in compressive or tensile stresses. Micro fuel cells, 
fabricated with different geometries, are tested, and their performance analyzed.

Professor Nowick, in key publications, showed that the radii of dopant ions in $\mathrm{CeO}_{2}$ have an important impact on the activation energy of oxygen ion conductivity. [21] This finding was further confirmed by early atomistic computer simulations. [22] Chatzichristodoulou, et al. in the article entitled Size of oxide vacancies in fluorite and perovskite structured oxides [23] discuss the growing interest in the chemical expansion of oxides upon loss of oxygen. The overall expansion of the lattice due to the reduction process has been tied to the competition between the enlargement of cation radius due to the decrease in their oxidation state and the relaxation around the missing oxygen, the latter described as an effective decrease in oxide vacancy radius. The authors in this work demonstrate important trends including the effective increase in oxide vacancy radius in fluorites with increase in host cation radius and its decrease with increasing dopant cation radius. They also confirm that the difference in radius between the oxygen ion and the oxygen ion vacancy in perovkites $\left(\mathrm{ABO}_{3}\right.$ structure) is considerably smaller than in the fluorite structure presumably due to the restraining action of the A-O sublattice to dimensional changes in the $\mathrm{B}-\mathrm{O}$ sublattice. There is obvious interest in tying these observations back to Nowick's interests in correlating oxygen ion migration with dopant ion radius.

\section{References}

1. A.S. Nowick, The golden age of crystal physics. Annu. Rev. Mater. Sci. 26, 1-19 (1996)

2. A.S. Nowick, B.S. Berry, Anelastic relaxation in crystalline solids (Academic, New York, 1972), p. 677

3. A.S. Nowick, Crystal properties via group theory (Cambridge University Press, Cambridge, 2005), p. 248

4. D.N. Beshers, Arthur Stanley Nowick, an intellectual appreciation. Solid State Phenom. 184, 7-13 (2012). doi:10.4028/www.scientific. net/SSP.184.7

5. Special Symposium in Honor of Professor Arthur S. Nowick. J. Phys. Chem. Solids, 55 [12], 1375-1534 (1994)

6. K Funke \& R. D. Banhatti \& L. G. Badr \& D. M. Laughman \& H. Jain, Toward understanding the second universality-A journey inspired by Arthur Stanley Nowick, J. Electroceram. This issue, DOI 10.1007/s10832-014-9898-0

7. W.K. Lee, J.F. Liu, A.S. Nowick, Limiting behavior of ac conductivity in ionically conducting crystals and glasses: A new universality. Phys. Rev. Lett. 67, 1559-1561 (1991)

8. M. R. Díaz-Guillén \& M. A. Frechero \& J. A. Díaz-Guillén \& A. F. Fuentes \& C. León, Nearly constant loss in crystalline oxide-ion conductor $\mathrm{Gd}_{2} \mathrm{Zr}_{2} \mathrm{O}_{7}$, J. Electroceram. This issue, DOI 10.1007/ s10832-014-9907-3

9. A. S. Nowick, A. V. Vaysleyb, H. Jain and X. Lu, Ac conductivity of crystalline materials and glasses ascribed to ADWPs, in electrically based microstructural characterization, R. A. Gerhardt, S. R. Taylor and E. J. Garboczi, eds. Mat. Res. Soc. Symp. Proc. 411, 99-112 (1996).

10. A. Shaw \& B. Deb \& S. Kabi \& A. Ghosh, Ion dynamics in single and mixed former glasses: Correlation between microscopic lengths and network structure, J. Electroceram. This issue, DOI 10.1007/ s10832-014-9932-2

11. L. H. Omari \& S. Sayouri \& T. Lamcharfi \& L. Hajji, Study of dielectric relaxation and diffuseness character of sol-gel derived $\mathrm{Pb}_{1-\mathrm{x}} \mathrm{La}_{\mathrm{x}}\left(\mathrm{Fe}_{0.03} \mathrm{Ti}_{0.97}\right) \mathrm{O}_{3}$ ceramics synthesized at lower temperature for modern nano-technological devices, J. Electroceram. This issue, DOI 10.1007/s10832-014-9921-5

12. Yuzhe Liu and Guangping Zheng, Anelastic analyses on the relaxation of anti-ferroelectric states in $0.94 \mathrm{Bi}_{0.5} \mathrm{Na}_{0.5} \mathrm{TiO}_{3}-0.06 \mathrm{BaTiO}_{3}$ solid solutions under electric fields, J. Electroceram. This issue, DOI 10.1007/s10832-014-9926-0

13. J. Habasaki \& K. L. Ngai, Molecular dynamics study of heterogeneous dynamics in lithium disilicate crystal, J. Electroceram. This issue, DOI 10.1007/s10832-014-9897-1

14. C Saiyasombat \& H Jain, Effect of tin and gold on sodium ion movement inA. a sodium silicate glass, J. Electroceram. This issue, DOI 10.1007/s10832-014-9933-1

15. M. Bokova \& I. Alekseev \& E. Bychkov, ${ }^{204} \mathrm{Tl}$ tracer diffusion and conductivity in thallium germanium sulphide glasses over a wide composition range, J. Electroceram. This issue, DOI 10.1007/ s10832-014-9906-4

16. J. Maier, Nanoionics: size effects and storage in small systems, J. Electroceram. This issue, DOI 10.1007/s10832-013-9886-9

17. W Shen \& J Jiang \& J. L. Hertz, Using thin films to investigate heterogeneous defect chemistry, J. Electroceram. This issue, DOI 10.1007/s10832-014-9931-3

18. P. Knauth \& J. Engel \& S. R. Bishop \& H. L. Tuller, Study of compaction and sintering of nanosized oxide powders by in situ electrical measurements and dilatometry: Nano $\mathrm{CeO}_{2}$ — case study, J. Electroceram. This issue, DOI 10.1007/s10832-014-9946-9

19. J.L. Hertz, H.L. Tuller, Micro-fuel Cells, in Microfabricated Power Generation Devices: Design and Technology, ed. by A. Mitsos, P.I. Barton (Wiley-VCH, Weinheim, 2009), pp. 51-80

20. Kian Kerman, Siyabulela Xuza, Shriram Ramanathan, Free standing yttria-doped zirconia membranes: Geometrical effects on stability, J. Electroceram. This issue, DOI 10.1007/s10832014-9917-1

21. R. Gerhardt-Anderson, A.S. Nowick, Ionic conductivity of $\mathrm{CeO}_{2}$ with trivalent dopants of different ionic radii. Solid State Ionics $\mathbf{5}$, 547-550 (1981)

22. V. Butler, C.R.A. Catlow, B.E.F. Fender, J.H. Harding, Dopant ion radius and ionic conductivity in cerium dioxide. Solid State Ionics $\mathbf{8}$, 109-113 (1983)

23. Christodoulos Chatzichristodoulou, Poul Norby, Peter V. Hendriksen \& Mogens B. Mogensen, Size of oxide vacancies in fluorite and perovskite structured oxides, J. Electroceram. This issue, DOI 10. 1007/s10832-014-9916-2 